\title{
HOMOTOPICAL AND TOPOLOGICAL RIGIDITY OF HYPERSURFACES OF SPHERICAL SPACE FORMS
}

\author{
PEDRO ZÜHLKE
}

\begin{abstract}
The first main result is a topological rigidity theorem for complete immersed hypersurfaces of spherical space forms from which similar theorems due to Wang/Xia and Longa/Ripoll can be derived. Under certain sharp conditions on the principal curvatures of such a hypersurface $f: N^{n} \rightarrow M^{n+1}(n \geq 2)$, it asserts that the universal cover of $N$ must be diffeomorphic to the $n$-sphere $\mathbb{S}^{n}$, and provides an upper bound for the order of the fundamental group of $N$ in terms of that of $M$. In particular, if $M=\mathbb{S}^{n+1}$, then $N$ is diffeomorphic to $\mathbb{S}^{n}$ and either $f$ or its Gauss map is an embedding.

Let $J \subset(0, \pi)$ be any interval of length less than $\frac{\pi}{2}$. The second main result constructs a weak homotopy equivalence between the space of all complete immersed hypersurfaces of $M$ with principal curvatures in $\cot (J)$ and the twisted product of $\left(\Gamma \backslash \mathrm{SO}_{n+2}\right)$ and Diff $+\left(\mathbb{S}^{n}\right)$ by $\mathrm{SO}_{n+1}$, where $\Gamma$ is the fundamental group of $M$ regarded as a subgroup of $\mathrm{SO}_{n+2}$.

Relying on another rigidity criterion due to Wang/Xia, the third main result constructs a homotopy equivalence between the space of all complete immersed hypersurfaces of $\mathbb{S}^{n+1}$ whose Gauss maps have image contained in a strictly convex ball and the same twisted product, with $\Gamma$ the trivial group.
\end{abstract}

\section{INTRODUCTION}

Convention. Throughout the article, $n \geq 2$ is an integer and manifolds are implicitly assumed to be connected, oriented and smooth, i.e., of class $C^{\infty}$. Maps between manifolds are also assumed to be smooth, and sets of such maps are furnished with the $C^{\infty}$-topology.

Topological rigidity. A classical theorem of J. Hadamard [6] states that a closed surface in the euclidean space $\mathbb{E}^{3}$ whose Gaussian curvature does not vanish must be embedded as the boundary of a convex body, and in particular diffeomorphic to the 2 -sphere. The following is the analogue of Hadamard's theorem for hypersurfaces of the sphere $\mathbb{S}^{n+1}$ (with the standard round metric).

(0.1) Theorem (do Carmo/Warner, [4, thm. 1.1]). Let $N^{n}$ be closed and $f: N^{n} \rightarrow \mathbb{S}^{n+1}$ be an immersion. Suppose that all sectional curvatures of $f$ are $\geq 1$. Then $N$ is diffeomorphic to $\mathbb{S}^{n}, f$ is an embedding and $f(N)$ is either totally geodesic (i.e., a great hypersphere) or contained in an open hemisphere. In the latter case, $f(N)$ is the boundary of a convex body. ${ }^{1}$

Because it will be necessary to consider several immersions $f: N^{n} \rightarrow M^{n+1}$ from a given manifold $N$ to a given Riemannian manifold $M$ at once, our viewpoint will be that $N$ is in each case furnished with the corresponding induced metric. As above, this is reflected in the terminology in that we speak of, e.g., the principal curvatures of $f$ (not of $N$ ). By a hypersurface of $M$ is meant such an immersion, not necessarily an embedding.

2010 Mathematics Subject Classification. Primary: 58D10, 53C24. Secondary: 53C40, 53C42.

Key words and phrases. h-principle; hypersurface; immersion; normal curvature; principal curvature; rigidity; sphere.

${ }^{1}$ The original statement also includes the assertion that $f$ is geometrically rigid in the sense that if $\bar{f}: N^{n} \rightarrow \mathbb{S}^{n+1}$ is another immersion inducing the same metric on $N$ as $f$, then there exists $Q \in \mathrm{O}_{n+2}$ such that $\bar{f}=Q \circ f$. For a closely related theorem of wider scope, see [5]. 
(0.2) Definition (Gauss map, dual). The Gauss map $\nu=\nu_{f}: N^{n} \rightarrow T M^{n+1}$ of an immersion $f: N^{n} \rightarrow M^{n+1}$ is uniquely determined by the condition that for all $p \in N$, $\left(u_{1}, \ldots, u_{n}\right)$ is a positively oriented orthonormal frame in $T N_{p}$ if and only if

$$
\left(d f_{p}\left(u_{1}\right), \ldots, d f_{p}\left(u_{n}\right), \nu(p)\right)
$$

is a positively oriented orthonormal frame in $T M_{f(p)}$. By convention, $\left(u_{1}, \ldots, u_{n+1}\right)$ is positively oriented in $T \mathbb{S}_{p}^{n+1}$ if and only if $\left(u_{1}, \ldots, u_{n+1}, p\right)$ is positively oriented in $\mathbb{R}^{n+2}$. For $M$ a spherical space form, the dual of $f$ (which need not be an immersion) is the map

$$
f^{\star}: N^{n} \rightarrow M^{n+1}, \quad p \mapsto \exp _{f(p)}\left(\frac{\pi}{2} \nu(p)\right) .
$$

Thus when $M=\mathbb{S}^{n+1}, f^{\star}$ is simply the Gauss map $\nu$ of $f$ regarded as a map into $\mathbb{S}^{n+1}$, and both notations will be used.

(0.3) Definition (principal radius, $J(f)$ ). Recall that a hypersphere of metric radius $r$ in $\mathbb{S}^{n+1}$ has principal curvatures equal to $\pm \cot r$. A principal radius of a hypersurface $f$ of a spherical space form is an element $\rho$ of the circle $\mathbb{R}(\bmod \pi)$ such that $\cot \rho$ is a principal curvature of $f$. An interval of $\mathbb{R}(\bmod \pi)$ is a connected subset thereof. We denote by $J(f) \subset \mathbb{R}(\bmod \pi)$ a smallest interval which contains all principal radii of $f .^{2}$

For reasons which will be clarified later, the main results are formulated in terms of principal radii. The following can be regarded as an extension of part of (0.1), as well as of similar theorems due to Wang/Xia and Longa/Ripoll (see (1.7) and (1.8)).

(0.4) Theorem (topological rigidity in $\mathbb{S}^{n+1}$ ). Let $f: N^{n} \rightarrow \mathbb{S}^{n+1}$ be an immersion. Suppose that $N$ is complete (with respect to the metric induced by $f$ ) and length $(J(f))<\frac{\pi}{2}$. Then:

(a) $N$ is diffeomorphic to $\mathbb{S}^{n}$.

(b) If $J(f)$ does not contain $0(\bmod \pi)$, then $f$ is an embedding.

(c) If $J(f)$ does not contain $\frac{\pi}{2}(\bmod \pi)$, then the dual of $f$ is an embedding.

In particular, (a)-(c) hold if $N$ is closed and $J(f)$ is disjoint from $J(f)+\frac{\pi}{2}$.

Example. To compare the hypotheses of (0.1) and (0.4), let $N^{n}$ be closed and $f: N^{n} \rightarrow \mathbb{S}^{n+1}$ be an immersion. Then

$$
J(f) \subset\left(0, \frac{\pi}{2}\right] \quad(\bmod \pi) \quad \text { or } \quad J(f) \subset\left[\frac{\pi}{2}, \pi\right) \quad(\bmod \pi)
$$

if and only if the principal curvatures are all nonnegative or nonpositive, respectively. If either holds, then Gauss' equation implies that the sectional curvatures are $\geq 1$.

Conversely, if the sectional curvatures are all $\geq 1$, then (1) must hold by (0.1) together with Gauss' equation. This is clear if $f$ is totally geodesic. Otherwise, by convexity, for each $p \in N, f(N)$ lies wholly on the side of the tangent hypersphere at $f(p)$ to which $\nu(p)$ or $-\nu(p)$ points, and the sign is independent of $p$ by connectedness. Hence no two principal curvatures have opposite signs.

(0.5) Remarks. In terms of principal curvatures, the conditions in (0.4) mean the following:

(i) $J(f)$ has length less than $\frac{\pi}{2}$ and does not contain $0(\bmod \pi)$ if and only if all principal curvatures of $f$ lie in an interval $(a, b)$ with $a b>-1$ and $b \in[0,+\infty]$ (where $( \pm \infty) 0=0$ ). Thus part (b) applies only to hypersurfaces whose sectional curvatures are greater than some $c>0$, but for $c$ as close to 0 as desired.

(ii) $J(f)$ has length less than $\frac{\pi}{2}$ and does not contain $\frac{\pi}{2}(\bmod \pi)$ if and only if all principal curvatures of $f$ lie in $(-\infty, a) \cup(b,+\infty)$ with $a b<-1$ and $b \in(0,+\infty)$. Thus part (c) applies only to hypersurfaces of two types: those whose sectional curvatures are everywhere strictly greater than 1 , and those with the property that

\footnotetext{
${ }^{2}$ If the set of all principal radii is contained in a half-open interval of length $\frac{\pi}{2}$, then $J(f)$ is uniquely determined; otherwise, it may not be. However, we are only interested in the former case.
} 
at every point, some sectional curvature is negative. The type depends on whether all principal curvatures have the same sign or not.

(0.6) Example. A celebrated theorem of J.F. Adams [1, thm. 1.1] states that it is possible to define a smooth $k$-dimensional frame field over $\mathbb{S}^{n}$ if and only if $0 \leq k \leq \rho(n+1)-1$, where $\rho$ is the Radon-Hurwitz function. By orthogonal duality, $\mathbb{S}^{n}$ supports a smooth $k$-dimensional distribution if and only if it supports an $(n-k)$-dimensional distribution. Assume without loss of generality that $2 k \leq n$. In this case, the existence of a $k$-dimensional distribution is equivalent to the existence of a $k$-dimensional frame field over $\mathbb{S}^{n}[14$, thm. 27.16]. In summary, $\mathbb{S}^{n}$ supports a $p$-dimensional distribution $(0 \leq p \leq n)$ if and only if either $p<\rho(n+1)$ or $n-p<\rho(n+1)$.

Now suppose that the principal curvatures of a closed hypersurface $f: N^{n} \rightarrow \mathbb{S}^{n+1}$ lie in $\left(-\infty,-c^{-1}\right) \cup(c,+\infty)$ for some $c \in(0,+\infty)$, e.g., they are greater than 1 in absolute value. Let $0 \leq p \leq n$ be the number of positive principal curvatures at some (hence every) point of $N$. The corresponding principal directions define a $p$-dimensional distribution over $N$. But by (0.4), $N$ is diffeomorphic to $\mathbb{S}^{n}$. In particular, if $n$ is even, we conclude that either $p=0$ or $p=n$, so that both $f(N)$ and $f^{\star}(N)$ must be embedded as boundaries of convex bodies.

For contrast,

$$
f: \frac{1}{\sqrt{2}}\left(\mathbb{S}^{k} \times \mathbb{S}^{n-k}\right) \hookrightarrow \mathbb{S}^{n+1} \quad(k, n-k \geq 1)
$$

has \pm 1 for its principal curvatures, so that $J(f)=\left[\frac{\pi}{4}, \frac{3 \pi}{4}\right]$ or $\left[-\frac{\pi}{4}, \frac{\pi}{4}\right](\bmod \pi)$. Consideration of normal translates of the latter shows that given any interval $J$ of length greater than $\frac{\pi}{2}$, there exists a closed hypersurface with principal radii in $J$ which is not diffeomorphic to a sphere. Moreover, setting $k=1$ and pre-composing with a self-map of $\mathbb{S}^{1}$ of degree greater than 1 , one can arrange that neither $f$ nor $f^{\star}$ be injective. In this sense, (0.4) is sharp.

(0.7) Theorem (topological rigidity in space forms). Let $M^{n+1}$ be a spherical space form and $f: N^{n} \rightarrow M^{n+1}$ an immersion. Suppose that length $(J(f))<\frac{\pi}{2}$ and $N$ is complete (with the metric induced by $f$ ). Then the universal cover of $N$ is diffeomorphic to $\mathbb{S}^{n}$. Moreover:

(a) If $J(f)$ does not contain $0(\bmod \pi)$ and $m$ denotes the maximum number of preimages under $f$ of a point in $f(N)$, then $m\left|\pi_{1}(N)\right| \leq\left|\pi_{1}(M)\right|$.

(b) If $J(f)$ does not contain $\frac{\pi}{2}(\bmod \pi)$ and $m^{\star}$ denotes the maximum number of preimages under $f^{\star}$ of a point in $f^{\star}(N)$, then $m^{\star}\left|\pi_{1}(N)\right| \leq\left|\pi_{1}(M)\right|$.

(c) Let $\operatorname{pr}_{M}: \mathbb{S}^{n+1} \rightarrow M$ be the covering projection. If $f$ is an embedding and $k$ denotes the number of components of $\operatorname{pr}_{M}^{-1}(f(N)) \subset \mathbb{S}^{n+1}$, then $k\left|\pi_{1}(N)\right|=\left|\pi_{1}(M)\right|$.

Remark. Even if $M$ or $N$ is not orientable, one can still define unsigned principal curvatures $\kappa \in[0,+\infty)$ of an immersion $f: N^{n} \rightarrow M^{n+1}$ using local Gauss maps. Suppose that all of them satisfy $\kappa<1-\varepsilon$, or all satisfy $\kappa>1+\varepsilon$ (for some $\varepsilon>0$ ). It follows from our proof that (0.7) still applies in these two situations, because any lift $\tilde{f}: \tilde{N} \rightarrow \mathbb{S}^{n+1}$ of $f$ to the universal cover $\tilde{N}$ of $N$ has the property that length $(J(\tilde{f}))<\frac{\pi}{2}$.

With this in mind, (0.7) (c) implies that one can obtain the conclusion of [8, thm. 2] while omitting their hypothesis on the distance to the cut locus and assuming only completeness, instead of closedness. Indeed, their restriction on the principal curvatures immediately implies that they must be greater than $1+\varepsilon$ in absolute value. ${ }^{3}$

Examples. Let $N^{n}$ be closed and possibly nonorientable, and $f: N^{n} \rightarrow \mathbb{R P}^{n+1}$ be an immersion. If the unsigned principal curvatures are all less (resp. greater) than 1 , then $N$ must be diffeomorphic to $\mathbb{S}^{n}$ or $\mathbb{R} \mathbb{P}^{n}$. Moreover, if $f\left(\right.$ resp. $f^{\star}$ ) is not an embedding, then $N=\mathbb{S}^{n}$. Compare [8, cor. 1.1].

\footnotetext{
${ }^{3}$ It should be noted that unlike here, in [8] the term "hypersurface" stands for (the image of) an embedding, while an immersion is called an "immersed hypersurface".
} 
When $n$ is even, the only nontrivial group which acts freely on $\mathbb{S}^{n}$ is $\mathbb{Z} / 2 \mathbb{Z}$; thus, in the situation of (0.7), $N$ is diffeomorphic to $\mathbb{S}^{n}$ (or $\mathbb{R}^{n}$ if nonorientability is allowed, as above).

Homotopical rigidity. Let $f: \mathbb{S}^{n} \rightarrow \mathbb{S}^{n+1}$ be an immersion and suppose that there exists $c \in \mathbb{S}^{n+1}$ such that $f\left(\mathbb{S}^{n}\right)$ is contained in the open hemisphere determined by $c$. A canonical choice would be to take $c$ as the (circum)center of $f\left(\mathbb{S}^{n}\right) .{ }^{4}$ Choose any $Q_{f} \in \mathrm{SO}_{n+2}$ satisfying $Q_{f}\left(-e_{n+2}\right)=c$, and let $\pi$ denote central projection of the southern hemisphere onto the affine hyperplane $\mathbb{R}^{n+1} \times\{-1\}$, which we identify with $\mathbb{E}^{n+1}$. Define $\bar{f}=\pi \circ Q_{f}^{-1} \circ f$. With the conventions described in (0.2), it is readily proved that the principal curvatures of $f$ are positive if and only if those of $\bar{f}$ are negative. Now it is a consequence of [16, prop. 4.3] that the space of all immersions $\mathbb{S}^{n} \rightarrow \mathbb{E}^{n+1}$ having negative principal curvatures is homotopy equivalent to the group Diff $+\left(\mathbb{S}^{n}\right)$ of orientation-preserving diffeomorphisms of $\mathbb{S}^{n}$. A homotopy equivalence simply assigns to each $\bar{f}$ its Gauss map.

Roughly, this means that a locally convex immersion $f: \mathbb{S}^{n} \rightarrow \mathbb{S}^{n+1}$, i.e., one whose principal curvatures are positive, is uniquely determined, up to homotopy, by the following data: an open hemisphere containing its image and the Gauss map $g_{f}$ of its composition with central projection. The antipode of the center of such a hemisphere is recorded by the last column of $Q_{f}$; the remaining columns correspond to a choice of coordinate axes for the tangent space to $\mathbb{S}^{n+1}$ at this point. A change of axes affects $g_{f}$ accordingly.

This suggests that $f \mapsto\left[Q_{f}, g_{f}\right]$ yields a homotopy equivalence between the space of locally convex hypersurfaces of $\mathbb{S}^{n+1}$ and the twisted product $\mathrm{SO}_{n+2} \times \mathrm{SO}_{n+1}$ Diff $+\left(\mathbb{S}^{n}\right)$. Recall that the latter is the quotient of $\mathrm{SO}_{n+2} \times \mathrm{Diff}_{+}\left(\mathbb{S}^{n}\right)$ under the equivalence relation which identifies $(Q, g)$ with $\left(Q P, P^{-1} g\right)$ for any $Q \in \mathrm{SO}_{n+2}, P \in \mathrm{SO}_{n+1}$ and $g \in \operatorname{Diff}_{+}\left(\mathbb{S}^{n}\right)$.

In fact, a similar weak homotopy equivalence $(w . h . e$.$) holds true for a more general class$ of spaces. However, for these it is simpler to construct the w.h.e. in the opposite direction.

(0.8) Definition $(\mathcal{F}(M ; I))$. Let $M^{n+1}$ be a Riemannian manifold and $I$ be any interval of the real line. The set of immersions $\mathbb{S}^{n} \rightarrow M^{n+1}$ whose principal curvatures take on values in $I$, equipped with the $C^{\infty}$-topology, will be denoted by $\mathcal{F}(M ; I)$.

Suppose $M$ is a spherical space form and $J \subset \mathbb{R}(\bmod \pi)$ is an interval of length less than $\frac{\pi}{2}$. Then it is reasonable to interpret $\mathcal{F}(M ; \cot J)$ as the space of all complete hypersurfaces of $M$ with principal curvatures in $\cot J$. This is clear when $M=\mathbb{S}^{n+1}$ due to (0.4). In the general case, one must identify two hypersurfaces which differ by a covering map; see (4.1). (0.9) Theorem (homotopical rigidity in $\left.\mathbb{S}^{n+1}, \mathrm{I}\right)$. Let $J \subset(0, \pi)$ be an interval such that $J \cap\left(J+\frac{\pi}{2}\right)=\emptyset$. Let $r \in J$ be arbitrary and $\iota_{r}: \mathbb{S}^{n} \rightarrow \mathbb{S}^{n+1}, p \mapsto \sin r p-\cos r e_{n+2}$. Then

$$
\Psi: \mathrm{SO}_{n+2} \times \mathrm{SO}_{n+1} \operatorname{Diff}_{+}\left(\mathbb{S}^{n}\right) \rightarrow \mathcal{F}\left(\mathbb{S}^{n+1} ; \cot J\right), \quad[Q, g] \mapsto Q \circ \iota_{r} \circ g
$$

is a weak homotopy equivalence.

(0.10) Remark. The group Diff $+\left(\mathbb{S}^{n}\right)$ is generally disconnected. The number of components is finite for all $n \neq 4$, and for $n \geq 5$ it coincides with the order of the group $\Theta_{n+1}$ of exotic spheres in dimension $n+1$ (see [3] and [9]). It follows that if $J$ contains a multiple of $\pi$ in its interior, then $\mathcal{F}\left(\mathbb{S}^{n+1} ; \cot J\right)$ cannot have the same homotopy type as the twisted product. For in this case the former has at least twice as many path-components as the latter. More precisely, the number of positive principal curvatures separates $\mathcal{F}\left(\mathbb{S}^{n+1} ; \cot J\right)$ into closedopen subspaces, and the subspace of hypersurfaces with positive (resp. negative) principal curvatures is weakly homotopy equivalent to $\mathrm{SO}_{n+2} \times \mathrm{SO}_{n+1}$ Diff $+\left(\mathbb{S}^{n}\right)$ by the theorem. We do not know what the homotopy type of the remaining components is.

Examples. If $n=2$ or 6 , then the principal bundle

$$
\mathrm{SO}_{n+2} \rightarrow \mathbb{S}^{n+1} \approx \mathrm{SO}_{n+2} / \mathrm{SO}_{n+1}
$$

\footnotetext{
${ }^{4}$ See $[2$, ch. II.2] for the definition and basic properties of the center of bounded subsets of CAT $(\kappa)$ spaces.
} 
admits a cross-section $\sigma .{ }^{5}$ This provides an $\mathrm{SO}_{n+1}$-equivariant homeomorphism $\mathbb{S}^{n+1} \times$ $\mathrm{SO}_{n+1} \rightarrow \mathrm{SO}_{n+2}$, given by $(z, P) \mapsto \sigma(z) P$. Thus (2) can be simplified to

$$
\Psi: \mathbb{S}^{n+1} \times \operatorname{Diff}_{+}\left(\mathbb{S}^{n}\right) \rightarrow \mathcal{F}\left(\mathbb{S}^{n+1} ; \cot J\right), \quad(z, g) \mapsto \sigma(z) \circ \iota_{r} \circ g \quad(n=2,6) .
$$

Theorems due to S. Smale [13] and A. Hatcher [7] guarantee that the inclusion $\mathrm{SO}_{n+1} \hookrightarrow$ Diff $_{+}\left(\mathbb{S}^{n}\right)$ is a homotopy equivalence for $n=2$ and 3 , respectively. It follows that in these two cases the w.h.e. (2) may be simplified to

$$
\Psi: \mathrm{SO}_{n+2} \rightarrow \mathcal{F}\left(\mathbb{S}^{n+1} ; \cot J\right), \quad Q \mapsto Q \circ \iota_{r} \quad(n=2,3){ }^{6}
$$

It is easily checked that these simplifications are equivalent when $n=2$.

Let $I \subset \mathbb{R}$ be an arbitrary interval, $M^{n+1}$ an arbitrary Riemannian manifold and pr: $\tilde{M} \rightarrow$ $M$ a Riemannian covering map. It can be shown [16, lem. 5.7] that the induced map $\mathrm{pr}_{*}: \mathcal{F}(\tilde{M} ; I) \rightarrow \mathcal{F}(M ; I), f \mapsto$ prof is also a covering map. Moreover, if pr is regular, then so is $\mathrm{pr}_{*}$, and their automorphism groups are isomorphic via $\gamma \mapsto \gamma_{*}$. Together with (0.9), this yields the following result; for a stronger version, see (4.2).

(0.11) Theorem (homotopical rigidity in space forms). Let pr: $\mathbb{S}^{n+1} \rightarrow M^{n+1}$ be a Riemannian covering and $J \subset(0, \pi)$ an interval such that $J \cap\left(J+\frac{\pi}{2}\right)=\emptyset$. Let $r \in J$ be arbitrary and $\iota_{r}: \mathbb{S}^{n} \rightarrow \mathbb{S}^{n+1}, p \mapsto \sin r p-\cos r e_{n+2}$. Then

$$
\operatorname{pr}_{*} \circ \Psi: \mathrm{SO}_{n+2} \times \mathrm{SO}_{n+1} \operatorname{Diff}_{+}\left(\mathbb{S}^{n}\right) \rightarrow \mathcal{F}(M ; \cot J), \quad[Q, g] \mapsto \operatorname{pr} \circ Q \circ \iota_{r} \circ g
$$

induces isomorphisms between $k$-th homotopy groups for $k \neq 1$, and yields an exact sequence

$$
1 \longrightarrow \pi_{1}\left(\mathrm{SO}_{n+2} \times \mathrm{SO}_{n+1} \operatorname{Diff}_{+}\left(\mathbb{S}^{n}\right)\right) \stackrel{\left(\mathrm{pr}_{*} \circ \Psi\right.}{\longrightarrow} \pi_{1}(\mathcal{F}(M ; \cot J)) \longrightarrow \pi_{1}(M) \longrightarrow 1 .
$$

Remark. The theorem implies in particular that (3) induces a bijection between the respective sets of path-components. It follows that any complete hypersurface $\mathbb{S}^{n} \rightarrow M^{n+1}$ whose principal curvatures are constrained to $\cot (J)$ can be deformed, through hypersurfaces of the same type, to a "hypersphere" of $M$ (i.e., the image of some hypersphere of $\mathbb{S}^{n+1}$ under the covering projection defining $M$ ).

More homotopical rigidity. Another topological rigidity criterion due to Wang/Xia ([15, thm. 1.2]) asserts that a complete hypersurface of $\mathbb{S}^{n+1}$ whose dual has image contained in a metric ball of radius $<\frac{\pi}{2}$ must be diffeomorphic to $\mathbb{S}^{n}$. We show that in this case it is an embedding, and apply their result to prove the following analogue of (0.9) concerning the space $\mathcal{H}$ of all such hypersurfaces. In the statement $\tau$ denotes the "orthogonal projection"

$$
\tau: \mathbb{S}^{n+1} \backslash\left\{ \pm e_{n+2}\right\} \rightarrow \mathbb{S}^{n}, \quad p \mapsto \frac{p-\left\langle p, e_{n+2}\right\rangle e_{n+2}}{\left|p-\left\langle p, e_{n+2}\right\rangle e_{n+2}\right|} .
$$

(0.12) Theorem (homotopical rigidity II). Let $\iota: \mathbb{S}^{n} \hookrightarrow \mathbb{S}^{n+1}$ denote set inclusion and $\mathcal{H}$ be the space described above. Then:

$$
\bar{\Psi}: \mathrm{SO}_{n+2} \times \mathrm{SO}_{n+1} \operatorname{Diff}_{+}\left(\mathbb{S}^{n}\right) \rightarrow \mathcal{H}, \quad[Q, g] \mapsto Q \circ \iota \circ g
$$

is a homotopy equivalence. In fact, given $f \in \mathcal{H}$, let $c_{f} \in \mathbb{S}^{n+1}$ be the center of the image of its dual. Choose any $Q_{f} \in \mathrm{SO}_{n+2}$ with $Q_{f}\left(-e_{n+2}\right)=c_{f}$ and set $g_{f}=\tau \circ Q_{f}^{-1} \circ f$. $A$ homotopy inverse of (4) is defined by:

$$
\bar{\Phi}: \mathcal{H} \rightarrow \mathrm{SO}_{n+2} \times \mathrm{SO}_{n+1} \operatorname{Diff}_{+}\left(\mathbb{S}^{n}\right), \quad f \mapsto\left[Q_{f}, g_{f}\right] .
$$

\footnotetext{
${ }^{5} \mathrm{~A}$ section is readily constructed using quaternions and Cayley numbers, respectively; see [14, thm. 8.6].

${ }^{6}$ This assertion is not obvious because the homotopy inverse need not be $\mathrm{SO}_{n+1}$-equivariant. It is rather a consequence of the fact that the space of locally convex immersions in $\mathbb{E}^{n+1}$ is homotopy equivalent to $\operatorname{Diff}_{+}\left(\mathbb{S}^{n}\right) \simeq \mathrm{SO}_{n+1}(n=2,3)$, to which the proof of $(0.9)$ is ultimately reduced.
} 
The analogues for $n=1$ of the spaces studied here are more complicated. A complete description in the locally convex case is obtained in [11], and [12] contains partial results in the general case.

Outline of the sections. In $\S 1$ we compute the principal curvatures of the dual and of the normal translates of an arbitrary immersion $f: N^{n} \rightarrow \mathbb{S}^{n+1}$ in terms of those of $f$. This is combined with (0.1) to establish the topological rigidity theorem (0.4) for hypersurfaces of $\mathbb{S}^{n+1}$. From this we then derive the aforementioned related results of Wang/Xia and Longa/Ripoll. It is also shown that if $f$ is locally convex, then there exists an open hemisphere which contains the images of both $f$ and $f^{\star}$.

The purpose of $\S 2$ is to prove the homotopical rigidity theorem (0.9). First it is proven that the inclusion $\mathcal{F}\left(\mathbb{S}^{n+1} ; \cot J\right) \hookrightarrow \mathcal{F}\left(\mathbb{S}^{n+1} ; \cot J^{\prime}\right)$ is a weak homotopy equivalence for any pair of intervals $J \subset J^{\prime} \subset(0, \pi)$ with $J^{\prime}$ disjoint from $J^{\prime}+\frac{\pi}{2}$. The proof relies on certain Möbius transformations and the results of $\S 1$. Using this, (0.9) is reduced to the locally convex case, which is proven directly by closely following the sketch provided above.

In $\S 3,(0.12)$ is established. Even though its statement is similar to that of (0.9), its proof is considerably easier.

Finally, $\S 4$ contains a proof of $(0.7)$, a resulting interpretation of $\mathcal{F}(M ; I)$ as a space of what we call "irreducible hypersufaces", and a slightly stronger but more technical formulation of (0.11). At the end of the paper three related open problems are listed.

\section{TOPOLOGICAL Rigidity}

We begin by studying the properties of translates of a hypersurface in the direction of its Gauss map.

(1.1) Lemma (parallel immersions). Let $f: N^{n} \rightarrow \mathbb{S}^{n+1}$ be an immersion and $\nu_{f}$ its Gauss map. Given $r \in \mathbb{R}$, define $f_{r}: N^{n} \rightarrow \mathbb{S}^{n+1}$ by

$$
f_{r}(p)=\exp _{f(p)}\left(r \nu_{f}(p)\right)=\cos r f(p)+\sin r \nu_{f}(p) \quad(p \in N) .
$$

Then the following assertions hold:

(a) $f_{r}$ is an immersion if and only if $r(\bmod \pi)$ is not a principal radius of $f$.

(b) Suppose that $f_{r}$ is an immersion. Let $p \in N$ and let $l$ denote the number of principal radii $\rho \in(0, \pi)(\bmod \pi)$ of $f$ at $p$ such that

$$
\sin \rho \sin (\rho-r)<0 .
$$

Then $l$ is independent of $p$. Moreover, $u \in T N$ is a principal direction for $f$ associated to the principal radius $\rho(\bmod \pi)$ if and only if $u$ is a principal direction for $f_{r}$ associated to the principal radius $(-1)^{l}(\rho-r)(\bmod \pi)$.

(c) If $f_{r}$ is an immersion and $r^{\prime} \in \mathbb{R}$, then $f_{r+r^{\prime}}=\left(f_{r}\right)_{(-1)^{l} r^{\prime}}$.

Remark. Note that the expression in (6) is independent of the representative $\rho$ of $\rho(\bmod \pi)$, as expected, but its sign depends on the representative $r$ of $r(\bmod \pi)$.

Proof. Let $u$ be a principal direction for $f$ associated to the principal radius $\rho \in(0, \pi)$ $(\bmod \pi)$. Then

$$
d\left(f_{r}\right)(u)=(\cos r-\cot \rho \sin r) d f(u)=\frac{\sin (\rho-r)}{\sin \rho} d f(u) .
$$

This is a positive or negative multiple of $d f(u)$ according to whether $\sin \rho \sin (\rho-r)$ is positive or negative. In particular, it is a nonzero multiple if and only if $r \not \equiv \rho(\bmod \pi)$, proving (a). 
Suppose now that $f_{r}$ is an immersion. The number $l$ defined in (b) is independent of the chosen point $p \in N$ by connectedness of $N$. It follows directly from (7) and our definition (0.2) of the Gauss map that

$$
\nu_{f_{r}}=(-1)^{l}\left(\cos r \nu_{f}-\sin r f\right),
$$

so that

$$
d\left(\nu_{f_{r}}\right)(u)=(-1)^{l+1}(\sin r+\cos r \cot \rho) d f(u) .
$$

Combining this with (7), one deduces that

$$
-d\left(\nu_{f_{r}}\right)(u)=(-1)^{l} \cot (\rho-r) d\left(f_{r}\right)(u) .
$$

Thus, $u$ is a principal direction for $f_{r}$ associated to the principal radius $(-1)^{l}(\rho-r)$ $(\bmod \pi)$. The converse is obtained by applying the same argument to $f_{r}$ using the identity $f=\left(f_{r}\right)_{(-1)^{l+1} r}$, which is derived from (c). In turn, part (c) follows from a straightforward computation using (8).

(1.2) Corollary (curvature of the dual). Let $M^{n+1}$ be a spherical space form and let $f: N^{n} \rightarrow M^{n+1}$ be an immersion. Then its dual $f^{\star}: N \rightarrow M$ is an immersion if and only if 0 is not a principal curvature of $f$. Moreover, in this case:

(a) The dual of $f^{\star}$ is $(-1)^{l+1} f$, where $l$ is the number of positive principal curvatures of $f$ at any point of $N$, and by definition $+f=f$ and $-f$ is given by $p \mapsto \exp _{f(p)}\left(\pi \nu_{f}(p)\right)$.

(b) $u$ is a principal direction for $f$ associated to the principal curvature $\kappa$ if and only if $u$ is a principal direction for $f^{\star}$ associated to the principal curvature $(-1)^{l+1} \kappa^{-1}$.

(c) $J\left(f^{\star}\right) \equiv \frac{\pi}{2} \pm J(f)(\bmod \pi)$.

Proof. For $M=\mathbb{S}^{n+1}$, apply (1.1) and (8) in the case where $r=\frac{\pi}{2}$. The general case is reduced to this one by lifting $f$ to $\tilde{f}: \tilde{N} \rightarrow \mathbb{S}^{n+1}$, where $\tilde{N}$ is the universal cover of $N$, and noting that the dual of $\tilde{f}$ is a lift of the dual of $f$. (For a careful argument establishing the latter, see the third paragraph of the proof of (0.7)).

(1.3) Lemma. Let $N^{n}$ be closed and $f: N^{n} \rightarrow \mathbb{S}^{n+1}$ be an immersion. Define

$$
F: N^{n} \times\left[0, \frac{\pi}{2}\right] \rightarrow \mathbb{S}^{n+1} \quad \text { by } \quad(p, t) \mapsto \exp _{f(p)}(t \nu(p))=\cos t f(p)+\sin t f^{\star}(p) .
$$

If the principal curvatures of $f$ are all positive (resp. nonnegative), then the open (resp. closed) hemisphere determined by the center of $f(N)$ contains the image of $F$.

(1.4) Remark. The center of the osculating circle of a normal section to $f$ at $p$ is $\exp _{p}(\rho \nu(p))$, where $\rho$ is the radius of curvature of the normal section. Thus, a convenient geometric interpretation for $F$ is the following: The image of $N \times\left(0, \frac{\pi}{2}\right)$ under $F$ is the locus of all possible centers of osculating circles to normal sections of $f$, given the information that the principal curvatures of $f$ are positive but otherwise unrestricted.

Proof. Assume first that the principal curvatures are positive. Then $f(N)$ is the boundary of a strictly convex body $B$; cf. (0.1) and [4, lem. 2.2]. Therefore, $f(N) \backslash\{f(q)\}$ is contained in the open hemisphere determined by $\nu(q)$ for each $q \in N$. Equivalently,

$$
\left\langle f(p), f^{\star}(q)\right\rangle \geq 0 \text { for all } p, q \in N,
$$

with equality holding if and only if $p=q$. Let $c \in \mathbb{S}^{n+1}$ denote the center of $f(N)$. By convexity, $c$ lies in the interior of $B$, so it may be written as $c=a_{1} f\left(p_{1}\right)+a_{2} f\left(p_{2}\right)$ for some $a_{1}, a_{2}>0$ and $p_{1} \neq p_{2} \in N$. It follows from (9) that $\left\langle c, f^{\star}(q)\right\rangle>0$ for all $q \in N$. Consequently, the open hemisphere determined by $c$ contains both $f^{\star}(N)$ and $f(N)$, the latter by the definition of $c$. But being convex, this hemisphere must also contain the image of $F$.

In case the principal curvatures are only nonnegative, the assertion can be deduced from the preceding paragraph by regarding $f$ as the limit of $f_{r}$ as $r \rightarrow 0^{+}$. 
Proof of (0.4). Suppose first that $J(f)$ does not contain $0(\bmod \pi)$. Then by (0.5) (i) combined with the theorem of Bonnet-Myers, $N$ must be closed. Together with the hypothesis that length $(J(f))<\frac{\pi}{2}$, this in turn implies that $J(f)$ is contained in $\left(r, r+\frac{\pi}{2}\right)(\bmod \pi)$ for some $r \in\left(0, \frac{\pi}{2}\right)$. By $(1.1)(\mathrm{b})$,

$$
J\left(f_{r}\right) \equiv J(f)-r \subset\left(0, \frac{\pi}{2}\right) .
$$

In particular, (0.1) applied to $f_{r}$ guarantees that $N$ is diffeomorphic to $\mathbb{S}^{n}$, thus establishing (a) in this case. Moreover, by (1.1) (c), $f=\left(f_{r}\right)_{-r}$. Thus, to prove (b) it will be sufficient to show that: if $g: N^{n} \rightarrow \mathbb{S}^{n+1}$ is an immersion with $J(g) \subset\left(0, \frac{\pi}{2}\right)(\bmod \pi)$, then $g_{-r}$ is an embedding for all $r \in\left[0, \frac{\pi}{2}\right]$.

Let $B$ be the convex body bounded by $g(N)$. Because the principal curvatures of $g$ are positive (instead of negative),

$\nu_{g}$ points towards the interior of $B$ at every point of $N$.

Define

$$
s=\sup \left\{t \in\left[0, \frac{\pi}{2}\right]: g_{-r} \text { is an embedding for all } r \in[0, t]\right\} .
$$

Suppose for the sake of obtaining a contradiction that $s<\frac{\pi}{2}$. Recall that embeddings form an open subset of $C^{\infty}\left(N, \mathbb{S}^{n+1}\right)$. Moreover, $g_{-r}$ is an immersion for all $r \in\left[0, \frac{\pi}{2}\right]$ by (1.1) (a). Hence $g_{-s}$ cannot be injective. Let $p \neq q \in N$ be such that $g_{-s}(p)=g_{-s}(q)$. As we are in codimension $1, d\left(g_{-s}\right)\left(T N_{p}\right)$ and $d\left(g_{-s}\right)\left(T N_{q}\right)$ are not transverse, for otherwise there would exist $s^{\prime}<s$ such that $g_{-s^{\prime}}$ is likewise not injective. Thus $\nu_{g_{-s}}(p)= \pm \nu_{g_{-s}}(q)$ in addition to $g_{-s}(p)=g_{-s}(q)$. If $\nu_{g_{-s}}(p)=\nu_{g_{-s}}(q)$, then it follows immediately from the definition that

$$
g(p)=\left(g_{-s}\right)_{s}(p)=\left(g_{-s}\right)_{s}(q)=g(q) ;
$$

but this is impossible because $g$ is an embedding, by (0.1).

Hence $\nu_{g_{-s}}(p)=-\nu_{g_{-s}}(q)$. Let $C$ be the unique great circle tangent to these. Then $g(p)$ and $g(q)$ bound a segment $S \subset B$ of $C$ of length less than $\pi$, since $g(N)$ is contained in an open hemisphere. But by $(10), \nu_{g}(p)$ and $\nu_{g}(q)$ both point to the interior of $S$. Hence $g_{-s}(p)$ and $g_{-s}(q)$, which are obtained from $g(p)$ and $g(q)$ by moving a distance $s<\frac{\pi}{2}$ towards the exterior of $S$ along $C$, cannot be equal. This is a contradiction. Thus, if $J(f)$ does not contain $0(\bmod \pi)$, then $N$ is diffeomorphic to $\mathbb{S}^{n}$ and $f$ is an embedding.

Suppose now that $J(f)$ does not contain $\frac{\pi}{2}(\bmod \pi)$. Then, by $(1.2)(\mathrm{c}), J\left(f^{\star}\right) \equiv \frac{\pi}{2} \pm J(f)$ $(\bmod \pi)$ does not contain $0(\bmod \pi)$. Let $g$ and $g^{\star}$ denote the Riemannian metrics on $N$ induced by $f$ and $f^{\star}$, respectively. By (0.5) (ii) there exists $k>0$ such that the principal curvatures of $f$ are greater than $k$ in absolute value. Using (7) with $r=\frac{\pi}{2}$, it follows that

$$
g^{\star}(u, u) \geq k^{2} g(u, u)
$$

for any principal direction $u$. But by (1.2) (b), the principal directions of $f$ and $f^{\star}$ are the same. Since at every point it is possible to find an orthogonal basis (with respect to either metric) consisting of such directions, (11) holds for any tangent vector $u$. It follows that $\left(N, g^{\star}\right)$ is complete since $(N, g)$ is. Therefore $f^{\star}$ satisfies the hypotheses of part (b). By what was proven above, it must be an embedding and $N$ must be diffeomorphic to $\mathbb{S}^{n}$.

(1.5) Remark. The following fact established in the preceding paragraph will be invoked later: Under the hypotheses of (0.4) (c), $N$ is complete with respect to the metric induced by $f^{\star}$.

(1.6) Corollary. Let $f: N^{n} \rightarrow \mathbb{S}^{n+1}$ be a closed hypersurface whose sectional curvatures are all greater than 1. Then $f$ is homotopic, through embeddings, to either $f^{*}$ or $-f^{*}$.

Proof. The homotopy is provided by $t \mapsto f_{t}$ for $t \in\left[0, \frac{\pi}{2}\right]$ in case the principal curvatures are all negative, and by $t \mapsto f_{-t}$ otherwise. 
(1.7) Theorem (Wang/Xia, [15, thm. 1.1]). Let $N^{n}$ be a closed manifold and $f: N^{n} \rightarrow \mathbb{S}^{n+1}$ be an immersion. Suppose that $f(N)$ is contained in an open hemisphere and that the GaussKronecker curvature of $f$ does not vanish. Then $N$ is diffeomorphic to $\mathbb{S}^{n}$.

Proof. Comparison of $f(N)$ with its metric circumsphere (cf. [2, II.2.7]), shows that $N$ contains a point where the principal curvatures have the same sign. Since by hypothesis the Gauss-Kronecker curvature is nonvanishing and $N$ is connected, all principal curvatures must have the same sign. Thus $J(f) \subset\left(0, \frac{\pi}{2}\right)$ or $\left(\frac{\pi}{2}, \pi\right)(\bmod \pi)$, and $(0.4)(\mathrm{a}, \mathrm{b}, \mathrm{c})$ apply.

(1.8) Theorem (Longa/Ripoll, [8, thm. 1]). Let $N^{n}$ be closed and $f: N^{n} \rightarrow \mathbb{S}^{n+1}$ be an immersion. Let $r$ be the radius of the smallest closed metric ball containing $f(N)$. If each principal curvature $\kappa$ of $f$ satisfies $|\kappa|>\tan \left(\frac{r}{2}\right)$, then $N$ is diffeomorphic to $\mathbb{S}^{n}$.

Proof. If $r<\frac{\pi}{2}$, then $f(N)$ is contained in an open hemisphere and no principal curvature is zero, hence (1.7) applies. If $r \geq \frac{\pi}{2}$, then all principal curvatures are greater than 1 in absolute value. Equivalently, $J(f)$ is contained in $\left(-\frac{\pi}{4}, \frac{\pi}{4}\right)(\bmod \pi)$, so $(0.4)(\mathrm{a}, \mathrm{c})$ apply.

\section{2. Номоторical Rigidity}

The following elementary result will be used together with (1.3) to uniformly increase the principal curvatures of a locally convex immersion.

(2.1) Lemma. Let $C \subset \mathbb{S}^{n+1}$ be a circle of radius $<\frac{\pi}{2}$. Let $\sigma_{c}: \mathbb{S}^{n+1} \rightarrow \hat{\mathbb{R}}^{n+1}=\mathbb{R}^{n+1} \cup$ $\{\infty\}$ denote stereographic projection from $-c$ and $M_{s}(s \in(0,1])$ be the family of Möbius transformations defined by

$$
M_{s}: \mathbb{S}^{n+1} \rightarrow \mathbb{S}^{n+1}, \quad p \mapsto \sigma_{c}^{-1}\left(s \sigma_{c}(p)\right) .
$$

Then the radius of $M_{s}(C)$ decreases strictly (and converges to 0 ) as $s$ decreases to 0 if and only if the center of $C$ lies in the closed hemisphere determined by $c$.

Proof. No generality is lost in assuming that $c=e_{n+2}$. Recall that Möbius transformations map circles to circles. The pull-back of the round metric under $\sigma^{-1}=\sigma_{c}^{-1}$ is given by

$$
g_{x}=4\left(1+|x|^{2}\right)^{-2} \bar{g}_{x} \quad\left(x \in \mathbb{R}^{n+1}\right),
$$

where $\bar{g}$ is the euclidean metric of $\mathbb{R}^{n+1}$. Let $[p, q]$ be a diameter of $C$ whose extension contains $e_{n+2}$ and, without loss of generality, let $I \subset \hat{\mathbb{R}}=\hat{\mathbb{R}} e_{1}$ be its image under $\sigma$.

* If $\infty \in I$, then $C$ is contained in the open southern hemisphere. For $s \in(0,1]$ close to 1 , the length of $[p, q]=\left[p,-e_{n+2}\right] \cup\left[-e_{n+2}, q\right]$ increases strictly as $s$ decreases.

* If $0 \in I$, then $C$ is contained in the open northern hemisphere, and $I=[a, b]$ with $a b \leq 0$. The length of $[s a, s b]$ with respect to $g$ decreases strictly with $s \in(0,1]$ since it is the sum of those of $[s a, 0]$ and $[0, s b]$.

* If neither $\infty$ nor 0 lie in $I$, then $I=[a, b]$ with $a b>0$. A simple computation shows that the length of $[s a, s b]$ decreases strictly together with $s$ if and only if $a b \leq 1$. As reflection in the equator of $\mathbb{S}^{n+1}$ corresponds under stereographic projection to inversion of $\hat{\mathbb{R}}^{n+1}$ in $\mathbb{S}^{n}$, the latter condition is equivalent to the midpoint of $[p, q]$ (i.e., the center of $C$ ) lying inside the closed northern hemisphere.

The conclusion follows since $M_{s}([p, q])$ is a diameter of $M_{s}(C)$.

(2.2) Corollary. Let $N^{n}$ be closed, $f: N^{n} \rightarrow \mathbb{S}^{n+1}$ be a locally convex immersion and $c$ be the center of $f(N)$. For $M_{s}$ as in (12), let $\mu(s)$ be the smallest principal curvature of $M_{s} \circ f$. Then $\mu(s)$ increases strictly to $+\infty$ as $s \in(0,1]$ decreases to 0 .

Proof. Let $p \in N$ be arbitrary and $u \in T N_{p}$. Let $\eta$ be the corresponding normal section, i.e., the curve which is the intersection of $f(V)$ with the unique totally geodesic 2 -sphere $\Sigma$ tangent to $u$ and $\nu(p)$, where $V$ is a small neighborhood of $p$. Finally, let $C \subset \Sigma$ be the unique 
osculating circle to $\eta$ at $f(p)$. Then $M_{s}(C)$ is the osculating circle to the normal section of $M_{s} \circ f$ determined by $u$. By (1.3) and (1.4), the center of $C$ lies inside the hemisphere determined by $c$. Hence the radius of $M_{s}(C)$ decreases strictly to 0 with $s \in(0,1]$ by (2.1). This implies the conclusion of the lemma.

(2.3) Proposition. Let $J \subset J^{\prime} \subset(0, \pi)$ be nondegenerate intervals with $J^{\prime} \cap\left(J^{\prime}+\frac{\pi}{2}\right)=\emptyset$. Then the inclusion

$$
i: \mathcal{F}\left(\mathbb{S}^{n+1} ; \cot J\right) \hookrightarrow \mathcal{F}\left(\mathbb{S}^{n+1} ; \cot J^{\prime}\right)
$$

is a weak homotopy equivalence.

Proof. The proof will be broken into several steps.

Step 1: If $J=(a, b)$ and $J^{\prime}=[a, b)$ or $(a, b] \subset(0, \pi)$ (with $\left.b-a \leq \frac{\pi}{2}\right)$, then the inclusion

$$
\mathcal{F}\left(\mathbb{S}^{n+1} ; \cot J\right) \hookrightarrow \mathcal{F}\left(\mathbb{S}^{n+1} ; \cot J^{\prime}\right)
$$

is a weak homotopy equivalence.

By compactness of $\mathbb{D}^{k}$, given any map of pairs

$$
F:\left(\mathbb{D}^{k}, \mathbb{S}^{k-1}\right) \rightarrow\left(\mathcal{F}\left(\mathbb{S}^{n+1} ; \cot (a, b]\right), \mathcal{F}\left(\mathbb{S}^{n+1} ; \cot (a, b)\right)\right) \quad\left(k \in \mathbb{N}^{+}\right),
$$

there exists $\varepsilon>0$ such that the image of $F$ is actually contained in $\mathcal{F}\left(\mathbb{S}^{n+1} ; \cot (a+\varepsilon, b]\right)$. Consequently, by (1.1) (b), $H:(s, p) \mapsto F(p)_{s \varepsilon}$ defines a homotopy of pairs between $F=H_{0}$ and a map $H_{1}$ with image in

$$
\mathcal{F}\left(\mathbb{S}^{n+1} ; \cot (a, b-\varepsilon]\right) \subset \mathcal{F}\left(\mathbb{S}^{n+1} ; \cot (a, b)\right) .
$$

This establishes the triviality of the relevant relative homotopy groups. The proof for $[a, b)$ is analogous.

Step 2: It can be assumed that $J^{\prime}$ is open.

Indeed, suppose that we have proved the proposition in this special case. Let $J \subset J^{\prime}$ be as in the hypothesis, but with $J^{\prime}$ not necessarily open.

If length $\left(J^{\prime}\right)<\frac{\pi}{2}$, then we can find an open interval $J^{\prime \prime}$ with $J \subset J^{\prime} \subset J^{\prime \prime}$. Under our assumption the latter inclusion, as well as the inclusion $J \subset J^{\prime \prime}$, yield w.h.e. of the corresponding spaces, hence so does $J \subset J^{\prime}$.

If length $\left(J^{\prime}\right)=\frac{\pi}{2}$, then $J^{\prime}$ cannot be closed, since $J^{\prime} \cap\left(J^{\prime}+\frac{\pi}{2}\right)=\emptyset$. If it is open, there is nothing to prove. Assume then that $J^{\prime}$ is half-open, and let $I, I^{\prime}$ denote the interiors of $J, J^{\prime}$. Then in the following commutative diagram of inclusions:

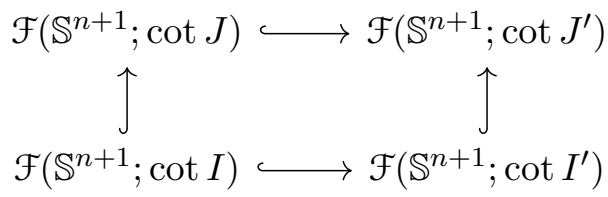

the bottom arrow is a w.h.e. as $I^{\prime}$ is open, and the arrow on the right is a w.h.e. by step 1 . If the length of $J$ is also $\frac{\pi}{2}$, then either $I=J$ or step 1 applies to the left vertical arrow; in either case, it is a w.h.e.. Finally, if the length of $J$ is less than $\frac{\pi}{2}$, then the left arrow is a w.h.e. by the preceding paragraph (with $I$ in place of $J$ and $J$ in place of $J^{\prime}$ ). Consequently, the top inclusion is also a w.h.e..

Step 3: It can be assumed that $J^{\prime} \subset\left(0, \frac{\pi}{2}\right)$.

By step 2, no generality is lost in assuming that $J^{\prime}$ is open, say, $J^{\prime}=(a, b)$. Let $\rho=\frac{a+b}{2}-\frac{\pi}{4}$. By (1.1) (b), $f \mapsto f_{\rho}$ yields a homeomorphism of pairs

$$
\left(\mathcal{F}\left(\mathbb{S}^{n+1} ; \cot J^{\prime}\right), \mathcal{F}\left(\mathbb{S}^{n+1} ; \cot J\right)\right) \rightarrow\left(\mathcal{F}\left(\mathbb{S}^{n+1} ; \cot \left(J^{\prime}-\rho\right)\right), \mathcal{F}\left(\mathbb{S}^{n+1} ; \cot (J-\rho)\right)\right) .
$$

But $J^{\prime}-\rho \subset\left(0, \frac{\pi}{2}\right)$ by the hypothesis on $J^{\prime}$ and the choice of $\rho$.

Step 4: It can be assumed that $J^{\prime}=\left(0, \frac{\pi}{2}\right)$. 
For, suppose that $J \subset J^{\prime} \subset\left(0, \frac{\pi}{2}\right)$ as in step 3 . To prove that (13) is a w.h.e. , it is sufficient to prove that the inclusions $J \subset\left(0, \frac{\pi}{2}\right)$ and $J^{\prime} \subset\left(0, \frac{\pi}{2}\right)$ induce w.h.e. of the corresponding spaces, that is, it can be assumed that the interval defining the target is $\left(0, \frac{\pi}{2}\right)$, as claimed. Step 5: The proposition holds in case $J \subset J^{\prime}=\left(0, \frac{\pi}{2}\right)$ is not closed.

By step 1, if $J$ is half-open then it can be replaced with its interior; in other words, it can be assumed that $J=(a, b) \subset\left(0, \frac{\pi}{2}\right)=J^{\prime}$. Let $J_{t}=(a-t a, b-t a)$ and define

$$
h_{t}: \mathcal{F}\left(\mathbb{S}^{n+1} ; \cot J\right) \rightarrow \mathcal{F}\left(\mathbb{S}^{n+1} ; \cot J_{t}\right), \quad f \mapsto f_{t} \quad(t \in[0, a]) .
$$

By (1.1), each $h_{t}$ is a homeomorphism. Let

$$
i_{J_{t}}: \mathcal{F}\left(\mathbb{S}^{n+1} ; \cot J_{t}\right) \hookrightarrow \mathcal{F}\left(\mathbb{S}^{n+1} ;(0,+\infty)\right) .
$$

Then $i_{J_{0}}=i_{J_{0}} \circ h_{0} \simeq i_{J_{t}} \circ h_{t}$. Therefore, to show that $i_{J}=i_{J_{0}}$ is a weak homotopy equivalence, it is sufficient to prove that $i_{J_{1}}$ is one. For this, let

$$
F:\left(\mathbb{D}^{k}, \mathbb{S}^{k-1}\right) \rightarrow\left(\mathcal{F}\left(\mathbb{S}^{n+1} ;(0,+\infty)\right), \mathcal{F}\left(\mathbb{S}^{n+1} ; \cot (0, b-a)\right)\right) \quad\left(k \in \mathbb{N}^{+}\right)
$$

be continuous and set $f^{z}=F(z)\left(z \in \mathbb{D}^{k}\right)$. Let $c(z)$ denote the center of $f^{z}\left(\mathbb{S}^{n}\right)$. Define a homotopy

$$
H:(0,1] \times \mathbb{D}^{k} \rightarrow \mathcal{F}\left(\mathbb{S}^{n+1} ;(0,+\infty)\right) \text { by } H(s, z)=M_{s}^{z} \circ f^{z},
$$

where $M_{s}^{z}$ is given by (12) with $c=c(z)$. By $(2.2)$, the restriction of $H$ to $[\varepsilon, 1] \times \mathbb{D}^{k}$ is a homotopy of pairs, and for $\varepsilon>0$ sufficiently small, $H_{\varepsilon}\left(\mathbb{D}^{k}\right) \subset \mathcal{F}\left(\mathbb{S}^{n+1} ; \cot (0, b-a)\right)$. This establishes that $i_{J_{1}}$ is a weak homotopy equivalence.

Step 6: The proposition holds in case $J \subset J^{\prime}=\left(0, \frac{\pi}{2}\right)$ is closed.

Let $J=[a, b] \subset\left(0, \frac{\pi}{2}\right)$. By step 5 , it suffices to show that

$$
\mathcal{F}\left(\mathbb{S}^{n+1} ; \cot [a, b)\right) \hookrightarrow \mathcal{F}\left(\mathbb{S}^{n+1} ; \cot [a, b]\right)
$$

is a weak homotopy equivalence. Let

$$
F:\left(\mathbb{D}^{k}, \mathbb{S}^{k-1}\right) \rightarrow\left(\mathcal{F}\left(\mathbb{S}^{n+1} ; \cot [a, b]\right), \mathcal{F}\left(\mathbb{S}^{n+1} ; \cot [a, b)\right)\right) \quad\left(k \in \mathbb{N}^{+}\right)
$$

be continuous. Fix $s \in(0,1]$ for now and let $f^{z}=F(z)$ and $M_{s}^{z}$ be as above. Recalling the construction of normal translates in (5), define

$$
H_{s}:[0, a) \times \mathbb{D}^{k} \rightarrow \mathcal{F}\left(\mathbb{S}^{n+1} ;(0,+\infty)\right) \text { by } H_{s}(t, z)=\left(M_{s}^{z} \circ f_{t}^{z}\right)_{-t} .
$$

We would like to extend this definition to $[0, a] \times \mathbb{D}^{k}$; the problem is that, by (1.1) (a), $f_{a}^{z}$ is not immersive at points where some principal radius equals $a$. Intuitively, at such a point the corresponding principal curvature is $+\infty$. However, this can be circumvented.

By (8), the Gauss map of $f_{t}^{z}$ is given by

$$
\nu_{f_{t}^{z}}=\cos t \nu_{f}-\sin t f^{z} \quad\left(t \in[0, a), z \in \mathbb{D}^{k}\right) .
$$

Further, since Möbius transformations are conformal, the Gauss map of $M_{s}^{z} \circ f_{t}^{z}$ is given by

$$
\nu_{M_{s}^{z} \circ f_{t}^{z}}(p)=\frac{d\left(M_{s}^{z}\right)_{f_{t}^{z}(p)}\left(\nu_{f_{t}^{z}}(p)\right)}{\mid \text { numerator } \mid} \quad\left(t \in[0, a), z \in \mathbb{D}^{k}, p \in \mathbb{S}^{n}\right) .
$$

Even though strictly speaking $f_{a}^{z}$ need not have a Gauss map, the expression on the right side of (15) is still sensible (and smooth) for $t=a$. Hence, so is that on the right side of (16); note that the denominator does not vanish since $M_{s}^{z}$ is a diffeomorphism. It is thus possible to extend (14) to all of $[0, a] \times \mathbb{D}^{k}$ by comparing (5) and setting

$$
H_{s}(t, z)=\cos t\left(M_{s}^{z} \circ f_{t}^{z}\right)-\sin t\left(\nu_{M_{s}^{z} \circ f_{t}^{z}}\right) \quad\left(t \in[0, a], z \in \mathbb{D}^{k}\right) .
$$

Now $M_{1}^{z}=\mathrm{id}_{\mathbb{S}^{n+1}}$, hence $H_{1}(t, z)=f^{z}$ for all $(t, z) \in[0, a] \times \mathbb{D}^{k}$. Therefore, by compactness of the latter, there exists $\varepsilon>0$ such that $H_{s}(t, z)$ is an immersion for all $(s, t, z) \in$ $[1-\varepsilon, 1] \times[0, a] \times \mathbb{D}^{k}$. 
Let $s \in[1-\varepsilon, 1]$ and $z \in \mathbb{D}^{k}$ be arbitrary. Since the principal radii of $f_{t}^{z}$ take on values in $[a-t, b-t] \subset(0, b-t]$ for all $t \in[0, a)$ by hypothesis, those of $H_{s}(t, z)$ lie in $(t, b]$ by $(2.2)$. Hence, those of $H_{s}(a, z)$ take on values in $[a, b]$ by continuity. We claim that they actually take values in $[a, b)$ for $s<1$.

Given a curve $\gamma$ on $\mathbb{S}^{2}$, define $\gamma_{a}$ by $\gamma_{a}(\tau)=\cos a \gamma(\tau)+\sin a \nu_{\gamma}(\tau)$, where $\nu_{\gamma}$ is its normal unit vector. Note that if $\gamma$ parametrizes a circle of radius $r>a$, then $\gamma_{a}$ parametrizes a circle of radius $r-a$. Let $C$ be the osculating circle to the normal section of $f^{z}$ determined by an arbitrary tangent vector $u \in T \mathbb{S}^{n}$. Then $\tilde{C}=\left(M_{s}(C)\right)_{-a}$ is the osculating circle to the normal section of $H_{s}(a, z)$ determined by $u$. If the radius of $C$ is greater than $a$ and $s<1$, then the radius of $\tilde{C}$ is smaller than that of $C$ by (2.1), proving the claim.

We conclude that $G:(s, z) \mapsto H_{s}(a, z)$ defines a homotopy of pairs connecting $G_{1}=F$ and a map $G_{1-\varepsilon}$ with $G_{1-\varepsilon}\left(\mathbb{D}^{k}\right) \subset \mathcal{F}\left(\mathbb{S}^{n+1} ; \cot [a, b)\right)$. This completes the proof of step 6 .

The conclusion now follows from the combination of steps $4-6$.

(2.4) Proposition (homotopical rigidity of locally convex immersions). Let $r \in\left(0, \frac{\pi}{2}\right)$ and $\iota_{r}: \mathbb{S}^{n} \rightarrow \mathbb{S}^{n+1}, p \mapsto \sin r p-\cos r e_{n+2}$. Then

$$
\Psi: \mathrm{SO}_{n+2} \times \mathrm{SO}_{n+1} \operatorname{Diff}_{+}\left(\mathbb{S}^{n}\right) \rightarrow \mathcal{F}\left(\mathbb{S}^{n+1} ;(0,+\infty)\right), \quad[Q, g] \mapsto Q \circ \iota_{r} \circ g
$$

embeds its domain as a deformation retract.

Given $f \in \mathcal{F}\left(\mathbb{S}^{n+1} ;(0,+\infty)\right)$, let $c_{f} \in \mathbb{S}^{n+1}$ denote the center of $f\left(\mathbb{S}^{n}\right)$. Choose $Q_{f} \in \mathrm{SO}_{n+2}$ with $Q_{f}\left(-e_{n+2}\right)=c_{f}$ and let $g_{f}: \mathbb{S}^{n} \rightarrow \mathbb{S}^{n}$ be the Gauss map of $\pi \circ Q_{f}^{-1} \circ f$, where $\pi$ denotes central projection of the southern hemisphere onto $\mathbb{E}^{n+1} \equiv \mathbb{R}^{n+1} \times\{-1\}$. Then a homotopy inverse of $\Psi$ is:

$$
\Phi: \mathcal{F}\left(\mathbb{S}^{n+1} ;(0,+\infty)\right) \rightarrow \mathrm{SO}_{n+2} \times \mathrm{SO}_{n+1} \operatorname{Diff}_{+}\left(\mathbb{S}^{n}\right), \quad f \mapsto\left[Q_{f}, g_{f}\right] .
$$

Proof. The proof consists of five steps.

Step 1: $\Psi$ is well-defined and continuous.

By $(1.1)$ (b) applied to the canonical inclusion $\iota: \mathbb{S}^{n} \hookrightarrow \mathbb{S}^{n+1}, \iota_{r}$ has principal radii everywhere equal to $r$. Thus $\iota_{r}$ lies in $\mathcal{F}\left(\mathbb{S}^{n+1} ;(0,+\infty)\right)$, and so does $Q \circ \iota_{r} \circ g$ for any $Q \in \mathrm{SO}_{n+2}$ and $g \in \operatorname{Diff}_{+}\left(\mathbb{S}^{n}\right)$. Moreover, $\iota_{r}$ commutes with elements of $\mathrm{SO}_{n+1}$, hence $\Psi$ is well-defined; it is clear that it is continuous. Note also that $\Psi$ is, up to homotopy, independent of the choice of $r$.

Step 2: $\Phi$ is well-defined and continuous.

Although $c_{f}$ is uniquely determined, $Q_{f}$, and hence also $\bar{f}=\pi \circ Q_{f}^{-1} \circ f$ and $g_{f}=\nu_{\bar{f}}$, are not. Nevertheless, any other choice $Q_{f}^{\prime} \in \mathrm{SO}_{n+2}$ is related to $Q_{f}$ by $Q_{f}^{\prime}=Q_{f} P$ for some $P \in \mathrm{SO}_{n+1}$. As $\pi$ commutes with elements of $\mathrm{SO}_{n+1}$, the corresponding $\bar{f}^{\prime}$ is given by

$$
\bar{f}^{\prime}=\pi \circ P^{-1} \circ Q_{f}^{-1} \circ f=P^{-1} \bar{f} .
$$

Therefore, the corresponding Gauss maps are related by

$$
g_{f}^{\prime}=\nu_{\bar{f}^{\prime}}=P^{-1} \circ \nu_{\bar{f}}=P^{-1} g_{f},
$$

so that $\left[Q_{f}, g_{f}\right]=\left[Q_{f}^{\prime}, g_{f}^{\prime}\right]$, that is, $\Phi$ is well-defined. It is also continuous because $c_{f}$ depends continuously on $f$ and the bundle projection $\mathrm{SO}_{n+2} \rightarrow \mathrm{SO}_{n+2} / \mathrm{SO}_{n+1} \approx \mathbb{S}^{n+1}$ admits local cross-sections.

Step 3: Suppose that the image of $h: \mathbb{S}^{n} \rightarrow \mathbb{S}^{n+1}$ is contained in the southern hemisphere. Then $h \in \mathcal{F}\left(\mathbb{S}^{n+1} ;(0,+\infty)\right)$ if and only if $\pi \circ h \in \mathcal{F}\left(\mathbb{E}^{n+1} ;(-\infty, 0)\right)$. In particular,

$$
\bar{f}=\pi \circ Q_{f}^{-1} \circ f \in \mathcal{F}\left(\mathbb{E}^{n+1} ;(-\infty, 0)\right) \text { and } g_{f} \in \operatorname{Diff}_{+}\left(\mathbb{S}^{n}\right) \text { if } f \in \mathcal{F}\left(\mathbb{S}^{n+1} ;(0,+\infty)\right) \text {. }
$$

It is clear that $h$ is an immersion if and only if $\pi \circ h$ is. By [4, prop. 2.3], the sectional curvatures of $h$ are everywhere greater than 1 if and only if those of $\pi \circ h$ are everywhere 
greater than 0. Equivalently through Gauss' equation, the principal curvatures of $h$ have the same sign everywhere if and only if the same holds for $\pi \circ h$. Because $\mathbb{S}^{n}$ is connected, it suffices to compute the sign at a single point. This will be done by comparison with an appropriate hypersphere. Let $\rho \in\left(0, \frac{\pi}{2}\right)$ be the radius of the smallest closed metric ball centered at $-e_{n+2}$ containing the image of $h$. Then $h$ and $\iota_{\rho}$ are tangent at any point $q \in \mathbb{S}^{n+1}$ where their images intersect. Further, these images lie on the same side of the great hypersphere tangent to them at $q$, namely, the one which contains $-e_{n+2}$. Thus, if the principal curvatures of $h$ are positive as those of $\iota_{\rho}$, then the Gauss maps of $h$ and $\iota_{\rho}$ coincide at (the corresponding preimages of) $q$. Hence so do those of $\pi \circ h$ and $\pi \circ \iota_{\rho}$ at $\pi(q)$. Moreover, $\pi \circ h\left(\mathbb{S}^{n}\right)$ is entirely contained in the closed ball bounded by $\pi \circ \iota_{\rho}\left(\mathbb{S}^{n}\right)$. Consequently their principal curvatures have the same sign at $\pi(q)$. But $\pi \circ \iota_{\rho}: p \mapsto \tan \rho p\left(p \in \mathbb{S}^{n}\right)$, and it is readily seen that the principal curvatures of the latter are negative, according to our convention (0.2). Finally, the Gauss map of an immersion into $\mathbb{E}^{n+1}$ having negative principal curvatures is an orientation-preserving diffeomorphism (see [16, lem. 4.2]).

Step 4: $\Phi \circ \Psi=\mathrm{id}$.

Let $Q \in \mathrm{SO}_{n+2}$ and $g \in \operatorname{Diff}_{+}\left(\mathbb{S}^{n}\right)$ be arbitrary. The center $c_{f}$ of the image of $f=$ $\Psi([Q, g])=Q \circ \iota_{r} \circ g$ is $Q\left(-e_{n+2}\right)$. Choose $Q_{f}=Q$ itself. Then

$$
\bar{f}=\pi \circ Q_{f}^{-1} \circ f=\pi \circ \iota_{r} \circ g=\tan r j \circ g,
$$

where $j: \mathbb{S}^{n} \hookrightarrow \mathbb{E}^{n+1}$ is the canonical inclusion. As $g$ preserves orientation,

$$
g_{f}=\nu_{\bar{f}}=\nu_{j \circ g}=\nu_{j} \circ g=\operatorname{id}_{\mathbb{S}^{n}} \circ g=g .
$$

Therefore $\Phi \circ \Psi([Q, g])=[Q, g]$.

Step 5: $\Psi \circ \Phi \simeq$ id.

Let $f \in \mathcal{F}\left(\mathbb{S}^{n+1} ;(0,+\infty)\right)$ be arbitrary and let $j_{r}: \mathbb{S}^{n} \rightarrow \mathbb{E}^{n+1}, p \mapsto \tan r p$. Then

$$
\Psi \circ \Phi(f)=Q_{f} \circ \iota_{r} \circ g_{f}=\left(Q_{f} \circ \pi^{-1}\right) \circ \pi \circ \iota_{r} \circ g_{f}=\left(Q_{f} \circ \pi^{-1}\right) \circ j_{r} \circ \nu_{\bar{f}} .
$$

Furthermore, we can write

$$
f=\left(Q_{f} \circ \pi^{-1}\right) \circ \pi \circ Q_{f}^{-1} \circ f=\left(Q_{f} \circ \pi^{-1}\right) \circ \bar{f} .
$$

Thus, by step 3, it suffices to construct a homotopy

$$
H:[0,1] \times \mathcal{F}\left(\mathbb{E}^{n+1} ;(-\infty, 0)\right) \rightarrow \mathcal{F}\left(\mathbb{E}^{n+1} ;(-\infty, 0)\right)
$$

such that $H_{0}(\phi)=\phi$ and $H_{1}(\phi)=j_{r} \circ \nu_{\phi}$ for any $\phi: \mathcal{F}\left(\mathbb{E}^{n+1} ;(-\infty, 0)\right)$. It is shown in [16, prop. 4.3] that the most natural homotopy $H:(s, \phi) \mapsto(1-s) \phi+s\left(j_{r} \circ \nu_{\phi}\right)$ works.

It is clear that $\Psi$ is an embedding onto a closed subspace. Moreover, for $r=\frac{\pi}{4}$, any element of the form $j_{r} \circ g$ (where $g \in \operatorname{Diff}_{+}\left(\mathbb{S}^{n}\right)$ ) is stationary under $H$. It follows that steps 4 and 5 yield a deformation retraction of $\mathcal{F}\left(\mathbb{S}^{n+1} ;(0,+\infty)\right)$ onto the image of $\Psi$.

Proof of (0.9). It can be assumed that $J$ is nondegenerate, for otherwise the assertion is almost trivial. By (1.1) (b), for $\rho \in(0, \pi)$ such that $\rho<J, f \mapsto f_{\rho}$ defines a homeomorphism

$$
h: \mathcal{F}\left(\mathbb{S}^{n+1} ; \cot J\right) \rightarrow \mathcal{F}\left(\mathbb{S}^{n+1} ; \cot (J-\rho)\right) .
$$

Furthermore, $h$ is compatible with $\Psi$ in the sense that if we denote by $\Psi_{r}$ the map given by (2), then $h \circ \Psi_{r}=\Psi_{r-\rho}$. Indeed, for any $Q \in \mathrm{SO}_{n+2}$ and $g \in \operatorname{Diff}_{+}\left(\mathbb{S}^{n}\right)$,

$$
h \circ \Psi_{r}([Q, g])=h\left(Q \circ \iota_{r} \circ g\right)=Q \circ\left(\iota_{r}\right)_{\rho} \circ g=Q \circ \iota_{r-\rho} \circ g=\Psi_{r-\rho}([Q, g]) .
$$

Thus $\Psi_{r}$ is a w.h.e. with $\mathcal{F}\left(\mathbb{S}^{n+1} ; \cot J\right)$ if and only if $\Psi_{r-\rho}$ is a w.h.e. with $\mathcal{F}\left(\mathbb{S}^{n+1} ; \cot (J-\rho)\right)$. Choosing $\rho$ appropriately, one obtains a reduction to the case where $J \subset\left(0, \frac{\pi}{2}\right)$. Then in 
the following commutative triangle:

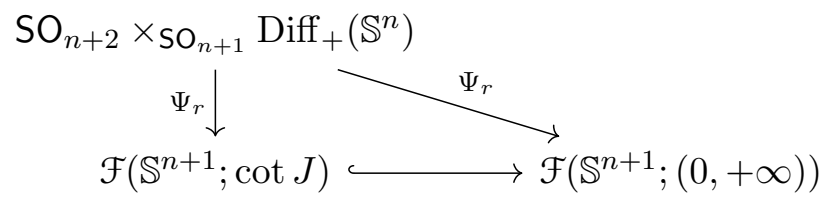

the diagonal arrow is a homotopy equivalence by (2.4), while (2.3) guarantees that the horizontal arrow is a weak homotopy equivalence. Therefore, so is the vertical arrow.

\section{Hypersurfaces Whose duals have image CONTAined in a hemisphere}

The purpose of this section is to provide a proof of (0.12).

(3.1) Definition. For each $s \in[0,1]$, let

$$
\zeta_{s}: \mathbb{S}^{n+1} \backslash\left\{ \pm e_{n+2}\right\} \rightarrow \mathbb{S}^{n+1} \backslash\left\{ \pm e_{n+2}\right\}, \quad q \mapsto \frac{q-s\left\langle q, e_{n+2}\right\rangle e_{n+2}}{\left|q-s\left\langle q, e_{n+2}\right\rangle e_{n+2}\right|} .
$$

Notice that $\zeta_{0}$ is the identity map. It will be convenient to regard $\zeta_{1}$ as a map into $\mathbb{S}^{n}$.

(3.2) Lemma. Let $q \in \mathbb{S}^{n+1}$ and $u, v \in T \mathbb{S}_{q}^{n+1}$ be mutually orthogonal unit vectors. Suppose that $\angle\left(v,-e_{n+2}\right) \leq r<\frac{\pi}{2}$. Then $\left|d\left(\zeta_{s}\right)_{q}(u)\right| \geq \cos r>0$ for any $s \in[0,1]$.

Proof. Notice first of all that $q$ must be distinct from $\pm e_{n+2}$ (i.e., $q$ belongs to the domain of $\zeta_{s}$ ), since it is orthogonal to $v$ by hypothesis. A straightforward computation yields that:

$$
d\left(\zeta_{s}\right)_{q}(u)=\frac{u-s\left\langle u, e_{n+2}\right\rangle e_{n+2}}{\left|q-s\left\langle q, e_{n+2}\right\rangle e_{n+2}\right|}+\frac{\left(2 s-s^{2}\right)\left\langle q, e_{n+2}\right\rangle\left\langle u, e_{n+2}\right\rangle}{\left|q-s\left\langle q, e_{n+2}\right\rangle e_{n+2}\right|^{3}}\left(q-s\left\langle q, e_{n+2}\right\rangle e_{n+2}\right) .
$$

After some labor, one obtains that:

$$
\left|d\left(\zeta_{s}\right)_{q}(u)\right|^{2}=\frac{1-\left(2 s-s^{2}\right)\left(\left\langle q, e_{n+2}\right\rangle^{2}+\left\langle u, e_{n+2}\right\rangle^{2}\right)}{\left[1-\left(2 s-s^{2}\right)\left\langle q, e_{n+2}\right\rangle^{2}\right]^{2}}
$$

Now for $s \in[0,1],\left(2 s-s^{2}\right)$ also lies in $[0,1]$, hence

$$
\left|d\left(\zeta_{s}\right)_{q}(u)\right|^{2} \geq 1-\left\langle q, e_{n+2}\right\rangle^{2}-\left\langle u, e_{n+2}\right\rangle^{2} \geq\left\langle v, e_{n+2}\right\rangle^{2} \geq \cos ^{2} r
$$

where the third inequality holds by hypothesis, while the second one follows from the fact that $e_{n+2}$ has norm 1 and $q, u, v$ are orthogonal unit vectors.

(3.3) Lemma. Let $N^{n}$ be a manifold and $h: N^{n} \rightarrow \mathbb{S}^{n+1}$ be an immersion which induces a complete Riemannian metric and whose Gauss map (dual) has image contained in the metric ball of radius $r<\frac{\pi}{2}$ about $-e_{n+2}$. Define $h_{s}=\zeta_{s} \circ h(s \in[0,1])$. Then:

(a) $h_{1}$ is an orientation-preserving diffeomorphism between $N^{n}$ and $\mathbb{S}^{n} .7$

(b) Each $h_{s}$, in particular $h=h_{0}$, is an embedding.

(c) The image of the Gauss map of $h_{s}$ is contained in the open hemisphere determined by $-e_{n+2}$ for each $s$.

Remark. The assertion that $h_{1}$ is a diffeomorphism under these hypotheses constitutes [15, thm. 1.2]. The proof presented here is the same as that given by Wang/Xia.

Proof of (3.3). Applying (3.2) with $q=h(p)$ and $v=\nu_{h}(p)$, one concludes immediately from the chain rule that

$$
\left|d\left(h_{s}\right)_{p}(u)\right| \geq \cos r\left|d h_{p}(u)\right| \quad \text { for all } p \in N, u \in T N_{p}, s \in[0,1] .
$$

Therefore $h_{s}$ is an immersion. In particular, $h_{1}: N^{n} \rightarrow \mathbb{S}^{n}$ is a local diffeomorphism. Since $N$ is complete with respect to the Riemannian metric $g$ induced by $h$, it is also complete with

\footnotetext{
${ }^{7}$ With the conventions adopted in (0.2).
} 
respect to the homothetic metric $\cos ^{2} r g$. But when $N$ is furnished with the latter, $h_{1}$ does not decrease distances, hence a standard argument (see [2, prop. I.3.28]) shows that $h_{1}$ is a covering map. As $\mathbb{S}^{n}$ is simply-connected, this in turn implies that $h_{1}$ is a diffeomorphism.

Suppose that $h_{s}(p)=h_{s}(q)$ for some $s \in[0,1)$ and $p, q \in N$. Taking the inner product of both sides with $e_{n+2}$, a straightforward computation shows that $\left\langle h(p), e_{n+2}\right\rangle=\left\langle h(q), e_{n+2}\right\rangle$, and this, together with $h_{s}(p)=h_{s}(q)$, in turn implies that $h(p)=h(q)$. But then clearly $h_{1}(p)=h_{1}(q)$, so that $p=q$ by the preceding paragraph. Thus each $h_{s}$ is an embedding by compactness of $N=\mathbb{S}^{n}$.

Finally, let $s \in[0,1], p \in N$ be arbitrary and $\left(u_{1}, \ldots, u_{n}\right)$ be a positively oriented frame in $T N_{p}$. Let $a \sim b$ indicate that $a$ is a positive multiple of $b$. Then:

$$
\begin{array}{rlrl} 
& \operatorname{det}\left(d\left(h_{s}\right)_{p}\left(u_{1}\right), \ldots, d\left(h_{s}\right)_{p}\left(u_{n}\right),-e_{n+2}, h_{s}(p)\right) & \\
\sim \operatorname{det}\left(d\left(h_{s}\right)_{p}\left(u_{1}\right), \ldots, d\left(h_{s}\right)_{p}\left(u_{n}\right),-e_{n+2}, h(p)\right) & & \left(\text { by the definition of } h_{s}\right) \\
\sim & \operatorname{det}\left(d h_{p}\left(u_{1}\right), \ldots, d h_{p}\left(u_{n}\right),-e_{n+2}, h(p)\right) & & \left(\text { by }(18), q=h(p), u=d h_{p}\left(u_{k}\right)\right) \\
\geq \cos r \operatorname{det}\left(d h_{p}\left(u_{1}\right), \ldots, d h_{p}\left(u_{n}\right), \nu_{h}(p), h(p)\right)>0 & & \left(\text { since } \angle\left(\nu_{h}(p),-e_{n+2}\right) \leq r<\frac{\pi}{2}\right)
\end{array}
$$

It follows that $\left\langle\nu_{h_{s}}(p),-e_{n+2}\right\rangle>0$, establishing (c). Moreover, as $h_{1}$ has image contained in $\mathbb{S}^{n} \subset \mathbb{R}^{n+1}$, by setting $s=1$ above, one deduces that $h_{1}$ is orientation-preserving.

(3.4) Definition. Let $\mathcal{H}$ denote the set of all immersions (or, equivalently by (3.3) (b), embeddings) of $\mathbb{S}^{n}$ into $\mathbb{S}^{n+1}$ whose Gauss maps have image contained in some open hemisphere depending upon the immersion, furnished with the $C^{\infty}$-topology.

Note that by [15, thm. 1.2] (or (3.3) (a)), $\mathcal{H}$ coincides with the space of all complete hypersurfaces of $\mathbb{S}^{n+1}$ whose Gauss maps have images contained in some strictly convex ball.

Proof of (0.12). If $P \in \mathrm{SO}_{n+1}$, then $P \circ \iota=\iota \circ P$. This implies that $\bar{\Psi}$ is well-defined. Similarly, $\bar{\Phi}$ is well-defined even though $Q_{f}$ and $g_{f}$ are not, because $\tau=\zeta_{1}$ commutes with elements of $\mathrm{SO}_{n+1}$, and any two choices of $Q_{f}$ differ by such an element. Furthermore, $\bar{\Phi}$ is continuous because $c_{f}$ depends continuously on $f$ and the bundle projection $\mathrm{SO}_{n+2} \rightarrow$ $\mathrm{SO}_{n+2} / \mathrm{SO}_{n+1} \approx \mathbb{S}^{n+1}$ admits local cross-sections. Further, $g_{f}$ is indeed an orientationpreserving diffeomorphism of $\mathbb{S}^{n}$ by (3.3) (a) applied to $h=Q_{f}^{-1} \circ f$.

Let $g \in \operatorname{Diff}_{+}\left(\mathbb{S}^{n}\right), Q \in \mathbb{S O}_{n+2}$ be arbitrary. The Gauss map of $\iota \circ g: \mathbb{S}^{n} \rightarrow \mathbb{S}^{n+1}$ is constant and equal to $-e_{n+2}$, as one verifies directly from the definition. Hence if

$$
f=Q \circ \iota \circ g=\bar{\Psi}([Q, g]),
$$

then we may choose $Q_{f}=Q$, so that $g_{f}=\tau \circ \iota \circ g=g$. Therefore $\bar{\Phi} \circ \bar{\Psi}$ is the identity map.

We claim that

$$
(s, f) \mapsto f_{s}=Q_{f} \circ \zeta_{s} \circ Q_{f}^{-1} \circ f \quad(s \in[0,1], f \in \mathcal{H})
$$

defines a homotopy connecting $\operatorname{id}_{\mathcal{H}}$ and $\bar{\Psi} \circ \bar{\Phi}$. It is clear that $f_{0}=f$ and $f_{1}=\bar{\Psi} \circ \bar{\Phi}(f)$ for any $f \in \mathcal{H}$. Moreover, taking $h=Q_{f}^{-1} \circ f$, it follows from (3.3) that each $f_{s}$ is an immersion (actually, an embedding) whose Gauss map has image contained in the open hemisphere determined by $c_{f}$. Thus $f_{s} \in \mathcal{H}$ for each $s$, and the proof is complete.

\section{Hypersurfaces of SPHERICAL SPACE FORMS}

The purpose of this section is to provide a proof of (0.7) and to justify the interpretation of $\mathcal{F}(M ; I)$ claimed in (0.8). Some related open problems are listed at the end. 
Proof of (0.7). Let $\operatorname{pr}_{N}: \tilde{N} \rightarrow N$ be the universal cover of $N$ and $\tilde{f}$ be any lift of $f$, so that

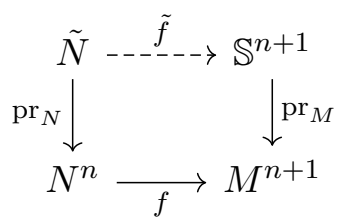

commutes. Since $\operatorname{pr}_{M}$ is a local isometry by hypothesis, the principal curvatures of $\tilde{f}$ and $f$ at corresponding points coincide; moreover, because $N$ is complete, so is $\tilde{N}$ (with respect to the metric induced by $\tilde{f}$ ). Hence $\tilde{f}$ satisfies the hypotheses of $(0.4)$, so that $\tilde{N}$ must be diffeomorphic to $\mathbb{S}^{n}$.

Suppose that $J(f)=J(\tilde{f})$ does not contain $0(\bmod \pi)$. Choose $q \in f(N)$ such that $\left|f^{-1}(q)\right|=m$ is as large as possible. Then, by commutativity of (19),

$$
m\left|\pi_{1}(N)\right|=\left|\operatorname{pr}_{N}^{-1} f^{-1}(q)\right|=\left|\tilde{f}^{-1} \operatorname{pr}_{M}^{-1}(q)\right| \leq\left|\operatorname{pr}_{M}^{-1}(q)\right|=\left|\pi_{1}(M)\right|,
$$

where the inequality comes from the fact that $\tilde{f}$ is injective, as guaranteed by (0.4) (b).

We claim that the dual of $\tilde{f}$ is a lift of the dual of $f$. To see this, express $N$ as the quotient of its universal cover $\tilde{N}$ by a free proper action of a group $H<\operatorname{Iso}_{+}(\tilde{N})$. Similarly, express $M$ as a quotient of $\mathbb{S}^{n+1}$ by some $G<\mathrm{SO}_{n+2}$. By connectedness of $N$ and commutativity of (19), for each $h \in H$ there exists $g \in G$ such that $\tilde{f} \circ h=g \circ \tilde{f}$. Therefore

$$
\nu_{\tilde{f}} \circ h=\nu_{\tilde{f} \circ h}=\nu_{g \circ \tilde{f}}=g \circ \nu_{\tilde{f}},
$$

where in the last equality the fact that $g \in \mathrm{SO}_{n+2}$ was used. As $\mathrm{pr}_{M}$ is a local isometry, $\exp _{M} \circ d\left(\operatorname{pr}_{M}\right)=\operatorname{pr}_{M} \circ \exp _{\mathbb{S}^{n+1}}$. Consequently $\tilde{f}^{\star}:=(\tilde{f})^{\star}$ factors through $N$, and its quotient is exactly $f^{\star}$. In other words,

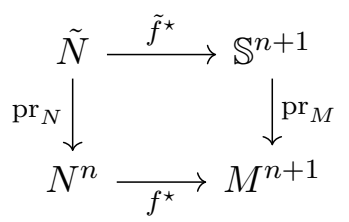

commutes, as claimed.

Suppose now that $J(f)$ does not contain $\frac{\pi}{2}(\bmod \pi)$. By (1.2) (b) (applied in the case where the ambient is $\left.\mathbb{S}^{n+1}\right)$,

$$
J\left(f^{\star}\right)=J\left(\tilde{f}^{\star}\right) \equiv \frac{\pi}{2} \pm J(\tilde{f}) \equiv \frac{\pi}{2} \pm J(f) \quad(\bmod \pi) .
$$

Moreover, $N$ is complete with respect to the metric induced by $f^{\star}$ because $\tilde{N}$ is complete with respect to the metric induced by $\tilde{f}^{\star}$ (by (1.5) applied to $\tilde{f}: \tilde{N} \rightarrow \mathbb{S}^{n+1}$ ). Thus $f^{\star}$ satisfies the hypotheses of (a), and this implies (b).

Suppose now that $f$ is an embedding. Then $\operatorname{pr}_{M}^{-1}(f(N))$ is an embedded submanifold of $\mathbb{S}^{n+1}$. Let $C$ be any of its connected components and $G_{C}=\{g \in G: g(C)=C\}$. The set inclusion $C \hookrightarrow \mathbb{S}^{n+1}$ has the same principal curvatures as $f$, hence $C$ must be diffeomorphic to $\mathbb{S}^{n}$ by $(0.4)$. As $f(N)$ is the quotient of $C$ by $G_{C}$,

$$
k\left|\pi_{1}(N)\right|=k\left|\pi_{1}(f(N))\right|=k\left|G_{C}\right|=|G|=\left|\pi_{1}(M)\right| .
$$

(4.1) Remark (irreducibility of hypersurfaces and $\mathcal{F}(M ; I)$ ). Let $N^{n}$ and $\bar{N}^{n}$ be arbitrary smooth manifolds and $M^{n+1}$ an arbitrary Riemannian manifold. Let us call a hypersurface $\bar{f}: \bar{N} \rightarrow M$ a factor of $f: N \rightarrow M$ if there exists a covering map pr: $N \rightarrow \bar{N}$ such that $f=\bar{f} \circ$ pr. Our viewpoint here is that $f$ and $\bar{f}$ are essentially the same object. Define $f$ to be irreducible if it has no factors arising from nontrivial covering maps. 
Now suppose that $N$ is closed, and furnish it with an arbitrary metric space structure compatible with its topology. If $f: N^{n} \rightarrow M^{n+1}$ is a hypersurface, then there exists $\varepsilon>0$ such that the restriction of $f$ to any ball of radius $\varepsilon$ is injective. Set

$$
G_{f}=\{\gamma \in \operatorname{Diff}(N): f \circ \gamma=f\} .
$$

The displacement function $p \mapsto d(p, \gamma(p))$ of an element $\gamma$ of $G_{f}$ cannot take on any positive value less than $\varepsilon$ by the choice of $\varepsilon$, hence $G_{f}$ acts freely on $N$. Moreover, if $N$ can be covered by $m$ balls of radius $\varepsilon$, then the order of $G_{f}$ is bounded above by $m$. We conclude that any hypersurface $f: N \rightarrow M$ has a unique irreducible factor, namely, that induced by $f$ on $N / G_{f}$.

Returning to our usual context, let $M^{n+1}$ be a spherical space form and $J \subset \mathbb{R}(\bmod \pi)$ be an interval of length $<\frac{\pi}{2}$. Then the preceding observation together with $(0.7)$ yield a bijective correspondence between $\mathcal{F}(M ; \cot J)$ and the set of all complete hypersurfaces of $M$ with principal curvatures in $\cot J$, modulo the equivalence relation that identifies two hypersurfaces if they have a common factor. The correspondence simply assigns to each element $f: \mathbb{S}^{n} \rightarrow M$ of $\mathcal{F}(M ; \cot J)$ (the equivalence class of) its unique irreducible factor.

(4.2) Remark (stronger version of (0.11)). Let $M^{n+1}$ be a spherical space form and $\Gamma$ its fundamental group. Then $\Gamma$ acts on $\mathbb{S}^{n+1}$ via deck transformations, which are isometries. Moreover, when regarded in this way as a subgroup of $\mathrm{SO}_{n+2}, \Gamma$ also acts naturally on $\mathrm{SO}_{n+2} \times \mathrm{SO}_{n+1}$ Diff $+\left(\mathbb{S}^{n}\right)$ through $\gamma[Q, g]=[\gamma Q, g]$. With respect to these actions, the w.h.e. $\Psi$ in $(0.9)$ becomes $\Gamma$-equivariant. Therefore, under the same hypotheses on $J$ as in that theorem, $\Psi$ induces a map

$$
\bar{\Psi}:\left(\Gamma \backslash \mathrm{SO}_{n+2}\right) \times \mathrm{SO}_{n+1} \operatorname{Diff}_{+}\left(\mathbb{S}^{n}\right) \rightarrow \mathcal{F}(M ; \cot J), \quad[\Gamma Q, g] \mapsto \operatorname{pr} \circ Q \circ \iota_{r} \circ g
$$

making the following square commutative, where the vertical arrows are covering maps:

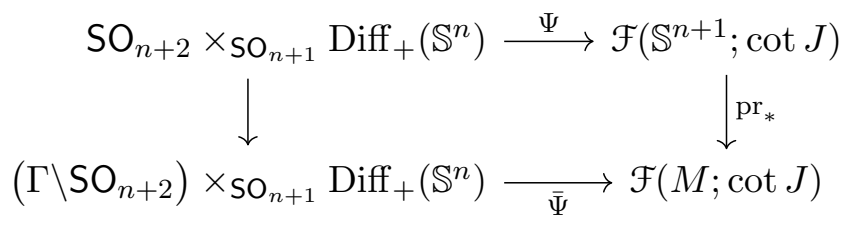

Looking at the short exact sequences relating the fundamental groups and applying the five-lemma, one deduces that $\bar{\Psi}$ must also be a w.h.e..

(1) Question. Let $J \subset(0, \pi)$ be an interval of length greater than $\frac{\pi}{2}$. Suppose that the closed manifold $N^{n}$ can be immersed in $\mathbb{S}^{n+1}$. Can it be immersed with principal curvatures in $\cot (J)$ ? More generally, is the inclusion of the space of immersions $N^{n} \rightarrow \mathbb{S}^{n+1}$ with principal curvatures in $\cot (J)$ into the space of all immersions a homotopy equivalence?

(2) Question. Let $J \subset \mathbb{R}(\bmod \pi)$ be an interval of length $<\frac{\pi}{2}$ containing $0(\bmod \pi)$. What is the homotopy type of $\mathcal{F}\left(\mathbb{S}^{n+1} ; \cot J\right)$ ? (Compare (0.6) and (0.10).)

In particular, is the existence of a $p$-dimensional distribution over $\mathbb{S}^{n}$ sufficient to guarantee the existence of a hypersurface in $\mathcal{F}\left(\mathbb{S}^{n+1} ; \cot J\right)$ with exactly $p$ positive principal curvatures at every point?

(3) Question. Let $n>2$ be odd. Then in the situation of (0.7), the theorem guarantees that $N^{n}$ has $\mathbb{S}^{n}$ for its universal cover. Must $N$ be diffeomorphic to a spherical space form? Compare [10].

Acknowledgements. The author thanks the Mathematics Department of the University of Brasília (Brazil) for hosting him as a post-doctoral fellow, and E. Longa and J. Ripoll for clarifications concerning [8]. Financial support by CAPES is gratefully acknowledged. 


\section{REFERENCES}

1. J. F. Adams, Vector fields on spheres, Ann. of Math. (2) 75 (1962), 603-632. 3

2. Martin Bridson and André Haefliger, Metric spaces of non-positive curvature, Grundlehren der mathematischen Wissenschaften, vol. 319, Springer-Verlag, Berlin, 1999. 4, 9, 15

3. Jean Cerf, La stratification naturelle des espaces de fonctions différentiables réelles et le théorème de la pseudo-isotopie, Inst. Hautes Études Sci. Publ. Math. (1970), no. 39, 5-173. 4

4. M. P. do Carmo and F. W. Warner, Rigidity and convexity of hypersurfaces in spheres, J. Differential Geometry 4 (1970), 133-144. 1, 7, 12

5. J.-H. Eschenburg, Local convexity and nonnegative curvature-Gromov's proof of the sphere theorem, Invent. Math. 84 (1986), no. 3, 507-522. 1

6. Jacques Hadamard, Sur certaines propriétés des trajectoires en dynamique, Journal de Mathématiques Pures et Appliquées 3 (1897), 331-388. 1

7. Allen Hatcher, A proof of the Smale conjecture, Diff $\left(S^{3}\right) \simeq \mathrm{O}(4)$, Ann. Math. 117 (1983), no. 3, 553-607. 5

8. Eduardo Longa and Jaime Ripoll, Topological rigidity for closed hypersurfaces of elliptic space forms, Preprint available at arxiv.org/abs/1801.07996v1, 2018. 3, 9, 17

9. John Milnor, Fifty years ago: topology of manifolds in the 50's and 60's, Low dimensional topology, IAS/Park City Math. Ser., vol. 15, Amer. Math. Soc., Providence, RI, 2009, pp. 9-20. 4

10. Ted Petrie, Free metacyclic group actions on homotopy spheres, Ann. of Math. (2) 94 (1971), 108-124. 17

11. Nicolau C. Saldanha, The homotopy type of spaces of locally convex curves in the sphere, Geom. Topol. 19 (2015), 1155-1203. 6

12. Nicolau C. Saldanha and Pedro Zühlke, On the components of spaces of curves on the 2-sphere with geodesic curvature in a prescribed interval, Internat. J. Math. 24 (2013), no. 14, 1-78. 6

13. Stephen Smale, Diffeomorphisms of the 2-sphere, Proc. Am. Math. Soc. 10 (1959), no. 4, 621-626. 5

14. Norman Steenrod, The topology of fibre bundles, Princeton University Press, Princeton, NJ, 1999. 3, 5

15. Qiaoling Wang and Changyu Xia, Rigidity of hypersurfaces in a Euclidean sphere, Proc. Edinb. Math. Soc. (2) 49 (2006), no. 1, 241-249. 5, 9, 14, 15

16. Pedro Zühlke, On a class of immersions of spheres into space forms of nonpositive curvature, Preprint available at arxiv.org/abs/1801.08524, 2018. 4, 5, 13

Departamento de Matemática, Universidade de Brasília (UNB)

Campus Darcy Ribeiro, 70910-900 - Brasília, DF, Brazil

E-mail address: zuehlkep@gmail.com 